\title{
The Role of Social Media during the COVID-19 Pandemic
}

\section{Dear Editor,}

With the widespread implementation of socialdistancing measures, the coronavirus disease (COVID-19) pandemic has resulted in an exponential increase in screen time for everyone. Personal and professional lives have merged through platforms such as Facebook, Twitter and Instagram, unified in isolation. By listing the ways in which social media has been used during the pandemic locally and globally, we hope to inspire readers to leverage on social media in employing novel methods in medical education, research, patient care and staff support. We also present potential pitfalls based on current literature.

\section{Spearheading Education and Research}

To circumvent the restrictions placed upon traditional face-to-face pedagogical methods, students and educators have been utilising social media for medical education. Twitter has been used to create global networks of information by facilitating academic forums and developing scholarly work through crowdsourcing. ${ }^{1}$ To solicit real time knowledge, the hashtag \#FOAMed (Free Open Access Medical Education) is also increasingly being used by the online medical community to collaborate and share best practices at a rate faster than large international organisations. ${ }^{1}$

Social media encourages brevity - it is commonly superseded by punchy tweets and visual-heavy content. By condensing daily statistics and evolving measures into nuggets of information that are easily disseminated to both the public and healthcare institutions, infographics effectively associate data with decreased cognitive load and stronger reader preferences. In Hong Kong, an infographic for airway management for suspected COVID-19 patients was distributed via Twitter and WeChat, supporting healthcare workers (HCWs) across multiple countries. ${ }^{2}$

Social media can also be used in the field of disease epidemiology. Twitter messages, particularly when location services are tagged can provide real-time approximations of disease prevalence without the delay of scheduled official releases. ${ }^{3}$ The trending of key words can reflect public concerns, giving authorities a sense of public sentiments. By mining data from Sino Weibo, public officials in Wuhan, China, were able to identify the elderly as a vulnerable population and subsequently directed the appropriate support resources. ${ }^{4}$ Any research in infectious disease in today's age of digital globalisation would be incomplete without tapping into the power of technology and social media for its development. ${ }^{5}$

Unfortunately, the qualities of social media such as its extensive penetrance and speed of information transmission have ironically led to the rapid proliferation of fake news through its various platforms. A recent study found that $27.5 \%$ of the most watched YouTube videos about COVID-19 included misinformation, reaching 62 million views globally. ${ }^{6}$ Such falsehood engender widespread public anxiety, life-threatening self-medication and non-compliance to COVID-19 measures. ${ }^{6}$ The influence of social media is so strong that the World Health Organisation has set up an Information Network for Epidemics aimed at tackling the "infodemic" by correcting and controlling the spread of false information. ${ }^{7}$ Measures include educating the public on indiscriminate dissemination of fake news as well as ensuring the availability of official sources of information to allow verification of online content.

\section{Patient Care and Staff Support}

The cessation of physical interactions during this pandemic has undermined how we practice patient-centred care, defined by in-person interaction. By tapping unto social media's diverse tools either in the form of audio or text, HCWs can facilitate virtual communication between isolated and sometimes dying patients with their loved ones, thereby comforting patients and supporting their bereaved family and friends. ${ }^{8}$ 
Social media can also provide emotional support for frontline HCWs who face significant psychological distress due to increased workload, social isolation, stigmatisation as well as concerns about being sources of infection to self and family members. ${ }^{9}$ In Wuhan, WeChat was used to provide support to frontline workers with issues of grief and burnout. ${ }^{10}$ Locally, senior management staff regularly spread messages of encouragement to all frontline workers within and beyond the healthcare sector via digital avenues (see Figure 1). Through regulated digital applications, social workers and therapists can also set up and promote peer support groups (see Figure 2). These platforms are important in creating safe spaces for $\mathrm{HCWs}$ to express strong emotions as they navigate complex issues of grief, uncertainties and ethical dilemmas relating to their duty of care to patients (see Figure 3). ${ }^{11}$ Through social media, members of the public are also able to express appreciation towards HCWs and dispel any misconceptions about them, strengthening solidarity among HCWs and the community.

However, despite its promises, social media remains an unfamiliar platform to $\mathrm{HCWs}$ who may report a lack of technical knowledge in accessing social media. ${ }^{12}$ Some HCWs perceive social media to be inefficient and a burden that takes away time and

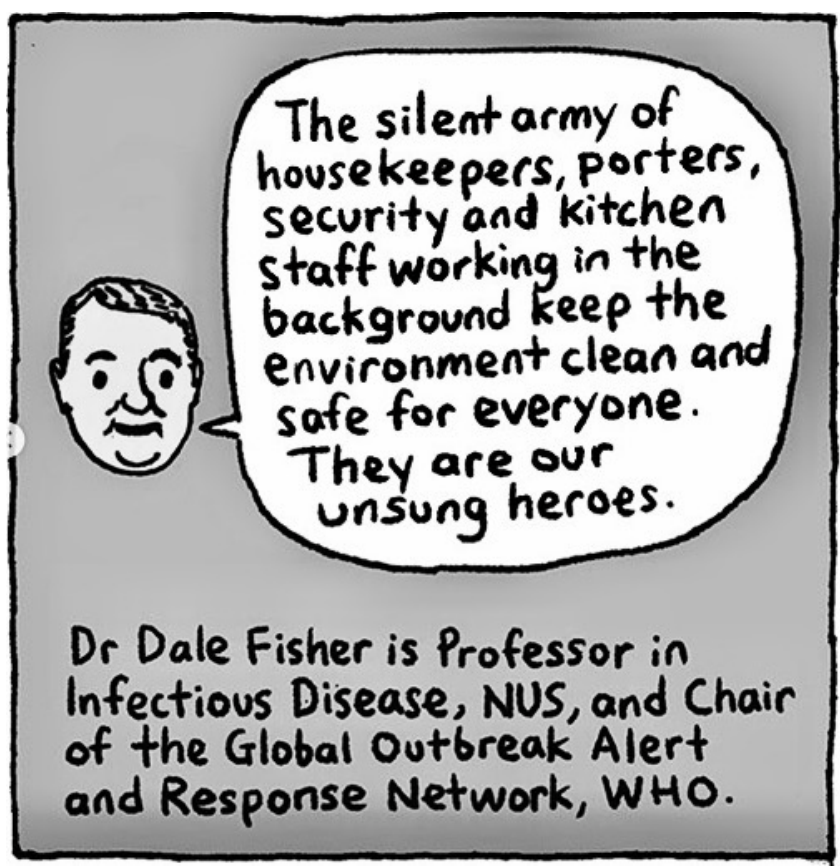

Fig. 1. An Instagram post showing appreciation to unsung heroes labouring during the COVID-19 pandemic.

(Reference: Nusmedicine. The COVID-19 Chronicles. Instagram; 2020)

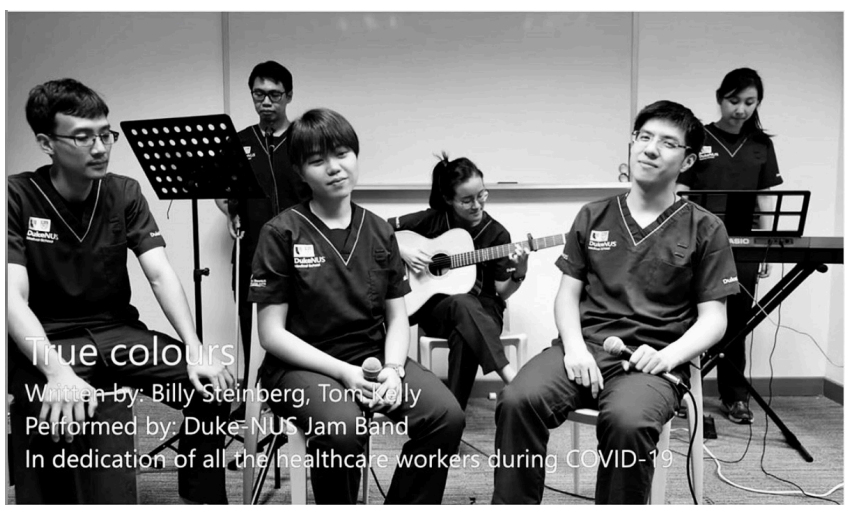

Fig. 2. Students from Duke-NUS Medical School encouraging the healthcare staff on the frontlines of COVID-19 via a video posted on Instagram.

(Reference: Singhealthmedicalhumanities. True colours. Instagram; 2020)

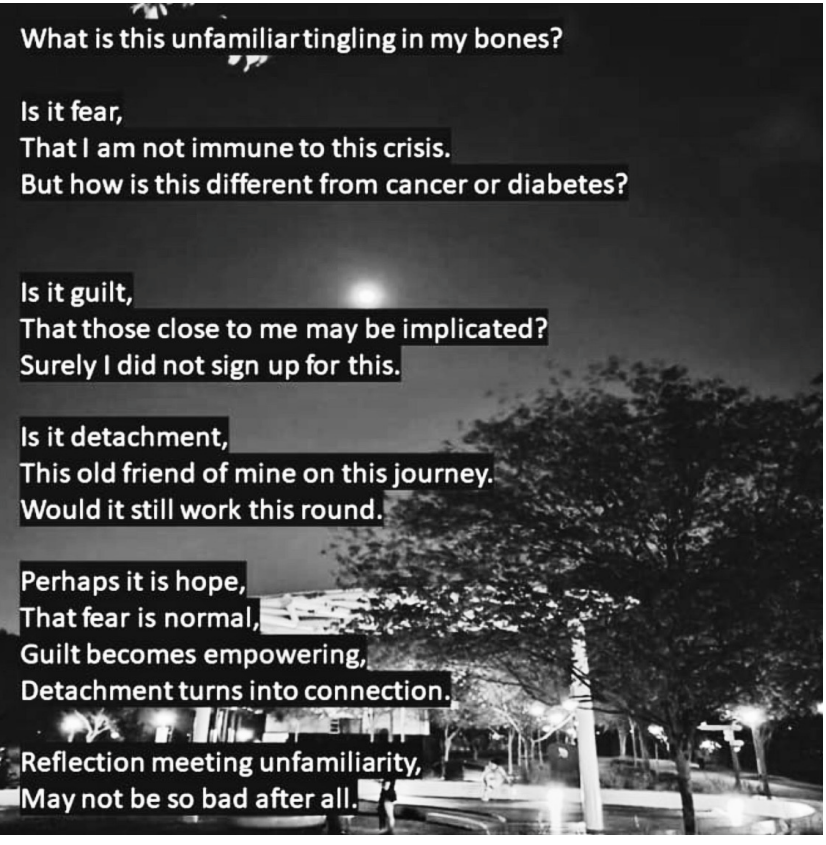

Fig. 3. An example of poetry expressing ineffable and heartfelt emotions during stressful periods on Instagram.

(Reference: Singhealthmedicalhumanities. Poem. 2020)

resources from their primary duties. Some also worry about damage to their professional image, with potential lawsuits for violations of personal and professional boundaries. ${ }^{13}$ An holistic collaboration between the respective stakeholders such as government bodies, hospital administrators, HCWs as well as various healthcare-related community groups is thus necessary to encourage and optimise the use of social media. In fact, given the borderless nature of such infectious disease outbreaks, an 
international effort of social media utilisation is also of utmost importance. ${ }^{14}$ Only when multiple levels of society see the relevance of social media and are unitedly reassured about its safety, can it gain traction to mitigate the adverse impacts of any healthcare crisis, especially one as contagious as COVID-19.

\section{Conclusion}

What is clearly emerging form this current pandemic is the imminent acceleration of information technology (IT) infrastructure development and digitalisation across all sectors worldwide. ${ }^{15}$ The potential of social media in healthcare is no longer something we can put aside; its systematic development, together with its utilisation for the good of our patients and fellow colleagues, is warranted.

\section{REFERENCES}

1. Rosenberg H, Syed S, Rezaie S. The Twitter pandemic: the critical role of Twitter in the dissemination of medical information and misinformation during the COVID-19 pandemic. CJEM 2020:1-4.

2. Chan AKM, Nickson CP, Rudolph JW, Lee A, Joynt GM. Social media for rapid knowledge dissemination: early experience from the COVID-19 pandemic. Anaesthesia 2020; doi.org/10.1111/ anae. 15057

3. Signorini A, Segre AM, Polgreen PM. The use of Twitter to track levels of disease activity and public concern in the U.S. during the influenza A H1N1 pandemic. PLoS One 2011;6:e19467; doi: 10.1371/ journal.pone.0019467.

4. Huang C, Xu X, Cai Y, Ge Q, Zeng G, Li X, et al. Mining the characteristics of COVID-19 patients in China: analysis of social media posts. J Med Internet Res 2020;22:e19087; doi: $10.2196 / 19087$

5. Wong SY, Tan BH. Megatrends in Infectious Diseases: the next 10 to 15 Years. Ann Acad Med Singapore. 2019;48:188-94.

6. Li HO, Bailey A, Huynh D, Chan J. YouTube as a source of information on COVID-19: a pandemic of misinformation? BMJ Glob Health 2020;5:e002604. doi: 10.1136/bmjgh-2020-002604.
7. Zarocostas J. How to fight an infodemic. The Lancet 2020;395:676.

8. Selman LE, Chao D, Sowden R, Marshall S, Chamberlain C, Koffman J. Bereavement support on the frontline of COVID-19: recommendations for hospital clinicians. J Pain Symptom Manage 2020:S0885-3924(20)30244-X; doi:10.1016/j. jpainsymman.2020.04.024.

9. Ho CS, Chee CY, Ho RC. Mental health strategies to combat the psychological impact of COVID-19 beyond paranoia and panic. Ann Acad Med Singapore 2020;49:155-60.

10. Cheng P, Xia G, Pang P, Wu B, Jiang W, Li Y-T, et al. COVID-19 epidemic peer support and crisis intervention via social media. Community Ment Health J. 2020;56:786-92.

11. Tai DY. SARS plague: duty of care or medical heroism? Ann Acad Med Singapore 2006;35:374-8.

12. Chan WS, Leung AY. Use of social network sites for communication among health professionals: systematic review. J Med Internet Res 2018;20:e117; doi: 10.2196/jmir.8382.

13. Ventola CL. Social media and health care professionals: benefits, risks, and best practices. P T. 2014;39:491-520.

14. Heymann DL. SARS and emerging infectious diseases: a challenge to place global solidarity above national sovereignty. Ann Acad Med Singapore 2006;35:350-3.

15. Contreras CM, Metzger GA, Beane JD, Dedhia PH, Ejaz A, Pawlik TM. Telemedicine: patient-provider clinical engagement during the COVID-19 pandemic and beyond. J Gastrointest Surg 2020:1-6.

Eng Koon Ong, ${ }^{1}{ }_{M B B S,}$ MMed, FAMS, Chu Hsien Lim, ${ }^{2}$ BSc Hons (Life Sciences), Alexandra JTY Wong, ${ }^{3} M B B S B S C$

${ }^{1}$ Division of Supportive and Palliative Care, National Cancer Centre Singapore, Singapore and Office of Medical Humanities, SingHealth Medicine Academic Clinical Programme, Singapore

${ }^{2}$ Duke-NUS Medical School, Singapore

${ }^{3}$ Emergency Department, Ng Teng Fong General Hospital, Singapore

Address for Correspondence: Ms Lim Chu Hsien, Duke-NUS Medical School, 8 College Road, Singapore 169857

Email: chuhsienlim@u.duke.nus.edu 